\title{
Efecto de la aplicación de un recubrimiento de almidón de yuca modificado por vía ácida sobre el tomate larga vida
}

\section{Effect of the application of an acid-modified cassava starch coating on the long-life tomato}

\author{
Nelsy Hoyos-Yela ${ }^{1}$; Rocío Pérez-Imbachíi ; Silvio Andrés Mosquera-Sánchez ; Sandra Patricia Paz-Peña ${ }^{4}$
}

'Ing. Agroindustrial. Universidad del Cauca. Popayán - Cauca, Colombia; e-mail: nelsyhoyos@hotmail.com; @https://orcid.org/0000-0002-9854-473X

${ }^{2}$ Ing. Agroindustrial. Universidad del Cauca. Popayán - Cauca, Colombia; e-mail: rocioperez283@gmail.com; @https://orcid.org/0000-0001-7913-6004

${ }^{3}$ Ing. Industrial de Alimentos. Universidad del Cauca. Popayán - Cauca, Colombia; e-mail: smosquera@unicauca.edu.co; Ohttps://orcid.org/0000-00022448-608X

${ }^{4}$ Ing. Agroindustrial. Universidad del Cauca. Popayán - Cauca, Colombia; e-mail: patty389@hotmail.com; Dhttps://orcid.org/0000-0002-7899-854X

Cómo citar: Hoyos-Yela, N.; Pérez-Imbachí, R.; Mosquera-Sánchez, S.A.; Paz-Peña, S.P. 2019. Efecto de la aplicación de un recubrimiento de almidón de yuca modificado por vía ácida sobre el tomate larga vida Rev. U.D.C.A Act. \& Div. Cient. 22(2):e1388. http://doi.org/10.31910/rudca.v22.n2.2019.1388

Artículo de acceso abierto publicado por Revista U.D.C.A Actualidad \& Divulgación Científica, bajo una licencia Creative Commons CC BY-NC 4.0

Recibido: Junio 8 de 2019

Aceptado: Noviembre 8 de 2019

Editado por: Rita María Ávila G. de Hernández

\section{RESUMEN}

Se buscó minimizar el efecto de factores ambientales sobre la calidad pos cosecha del tomate, mediante la aplicación de recubrimiento elaborado con almidón de yuca modificado por vía ácida. La modificación de la estructura original de una suspensión de almidón de yuca variedad SM 707-17, se realizó mediante adición de ácido cítrico, en concentración de $15 \% \mathrm{p} / \mathrm{p}$ respecto al almidón en base seca, bajo condiciones de temperatura y de velocidad de agitación controladas. El efecto de este recubrimiento, se comparó con el efecto de la aplicación de otros elaborados con base en almidón modificado enzimáticamente y almidón nativo. Se midieron las variables: sólidos solubles totales, firmeza, $\mathrm{pH}$, acidez titulable, tasa de respiración, pérdida de peso y color durante 15 días, aplicando un diseño factorial completamente al azar $4 \times 6$. Los resultados, se analizaron mediante el software SPSS versión 19, con el cual, se encontró que el factor "Recubrimiento" no influyó sobre las variables de respuesta analizadas, a diferencia del factor "Tiempo", que tuvo efecto decisivo sobre las mismas.
Palabras clave: Solanum lycopersicum; Manibot esculenta; poscosecha; revestimientos (Tesauro y Glosario Agrícola).

\section{ABSTRACT}

The aim was to minimize the effect of environmental factors on postharvest of tomato quality by applying a coating made with cassava starch modified by acid route. The modification of the original structure of a suspension of native starch of cassava variety SM 707-17, was carried out by addition of citric acid in concentration of $15 \% \mathrm{w} / \mathrm{w}$ with respect to the starch in dry base, under controlled temperature and speed of controlled agitation. The effect of this coating was compared with the effect of the application of others made on the basis of enzymatically modified starch and native starch. The variables total soluble solids, firmness, $\mathrm{pH}$, titrable acidity, respiration rate, weight loss and color for 15 days were measured, applying a completely randomized $4 \times 6$ factorial design. The results were analyzed using SPSS software version 19, which found that the "Coating" factor did not influence the 
response variables analyzed, unlike the "Time" factor that had a decisive effect on them.

Keywords: Solanum lycopersicum, Manihot esculenta Crantz: Postharvest; Cladding

\section{INTRODUCCIÓN}

El tomate es la hortaliza más consumida en Colombia, abasteciendo, aproximadamente, el 59\% de la población (Asohofrucol, 2014); su vida útil está entre 7 y 10 días almacenada, a $10-12,5^{\circ} \mathrm{C}$ y 90 , a 95\% HR, aunque tiende a deshidratarse por ser climatérico. La maduración está acompañada por cambios en el sabor, color, aroma, textura y pérdida de firmeza, debido a cambios físicos y químicos, asociados con la degradación de la pared celular y la solubilización de las pectinas por las enzimas pectinesterasa (PE), poligalacturonasa (PG) y pectatoliasa (PL) (San Martín et al. 2012).

En la actualidad, se está haciendo énfasis en el desarrollo de recubrimientos naturales comestibles para la conservación de productos frescos, que se caracterizan por ser biodegradables y amigables con el ambiente, al estar constituidos por materias primas renovables, en algunos casos, extraídas de subproductos de la agroindustria (Castillo et al. 2018).

El almidón es un ingrediente utilizado en la industria alimentaria como recubrimiento y, para impartir y mejorar propiedades funcionales. Las limitaciones del almidón nativo disminuyen su funcionalidad, debido al grado de ramificación, relación amilosa-amilopectina, distribución del peso molecular, condiciones de $\mathrm{pH}$, temperatura y presión, que alteran las propiedades mecánicas, térmicas, estructurales y de barrera, provocando baja resistencia a esfuerzos de corte, descomposición térmica, alto nivel de retrogradación y sinéresis (Bonilla et al. 2014), por lo que es conveniente la modificación de sus propiedades con el uso de productos químicos y biológicos o técnicas físicas (Gutiérrez et al. 2014), como reticulado (crosslinking), con fosfatos o adipatos, pre-gelatinización, dextrinación o hidrólisis ácida (Pérez, 2017). Para resaltar la importancia de su uso, se evaluó la respuesta fisiológica y estructural del tomate en poscosecha ante la aplicación de un recubrimiento obtenido por vía ácida, bajo condiciones ambientales.

\section{MATERIALES Y MÉTODOS}

Localización. El proyecto, se desarrolló en la Planta Piloto para el procesamiento de vegetales, Laboratorios de Biotecnología, Reología y Empaques de la Facultad de Ciencias Agrarias de la Universidad del Cauca y en la Unidad de Microscopía Electrónica del Museo de Historia Natural, en la ciudad de Popayán, Departamento del Cauca, Colombia, a una altitud de $1.738 \mathrm{~m}$ s.n.m., temperatura media de $19^{\circ} \mathrm{C}$ y humedad relativa (H.R) $77,75 \%$.

Tomate. Se usaron tomates larga vida en grado 4 de madurez, con diámetro ecuatorial promedio de $8 \mathrm{~cm}$ y axial de $6 \mathrm{~cm}$, variedad Indaba, producidos bajo cubierta y cultivados en la finca " $\mathrm{El}$ Porvenir”, ubicada en la vereda Cinco Días, del Municipio de Timbío
(Cauca), a una altura promedio de $1.850 \mathrm{~m}$ s.n.m., con temperatura media entre 16 y $23^{\circ} \mathrm{C}$.

Almidón de yuca. Se usó almidón nativo variedad SM 707-17, proveniente de la rallandería "La Agustina", ubicada en Mondomo (Cauca) y almidón de yuca SM 707-17, previamente modificado enzimáticamente con alpha - amylasa Bacillus amyloliquefaciens Ban 240L, con grado de modificación del 10\% de Equivalente de Dextrosa (E.D.) (Ordoñez et al. 2014).

Se usó ácido cítrico monohidrato grado analítico con pureza del 99,5\%, para la modificación del almidón de yuca por vía ácida; aceite esencial de limoncillo (Cymbopogon citratus) grado alimentario, por sus propiedades antimicrobianas y antibacteriales (Alzate et al. 2009); glicerina, como plastificante no volátil de bajo peso molecular, que mejora la flexibilidad, la manejabilidad y la habilidad de extensión del recubrimiento (Sánchez et al. 2015) y Tween 80, por su acción surfactante, para facilitar o aumentar la emulsividad, la dispersión y la adherencia del recubrimiento (Riojas et al. 2011).

Modificación del almidón con ácido cítrico. Se empleó una suspensión de $100 \mathrm{~g}$ de almidón al 40\% p/p B.S.; se hidrató por 1 h, se ajustó el pH hasta 5,5, se agitó a $175 \mathrm{rpm}$ y $52^{\circ} \mathrm{C}$ en shaker; se adicionó ácido cítrico y se agitó por $14 \mathrm{~h}$, luego, de lo cual se ajustó nuevamente el pH a 5,5. Se centrifugó el almidón a 4500rpm por $30 \mathrm{~min}$, se obtuvo el sobrenadante, con el que se midió el E.D en el espectrofotómetro (Bonilla et al. 2014).

Caracterización reológica de las suspensiones de almidón. Se prepararon $25 \mathrm{~mL}$ de suspensión al $8 \%$ B.S. en agua destilada (ICC Standard Method No. 162, 1995), con una muestra en polvo, con tamaño de partícula menor o igual a $250 \mu \mathrm{m}$, libre de partículas gruesas contaminantes, a 12\% de humedad, para el almidón nativo y para el almidón modificado. Le mezcla, se agitó y se introdujo en celda de empastamiento de almidón de un Reómetro Rotacional (TA Instruments, modelo $1500 \mathrm{EX}$ ), usando una rampa de calentamiento, desde $50^{\circ} \mathrm{C}$, a una velocidad de $12,2 \mathrm{C} / \mathrm{min}$ y velocidad angular, de $16,75 \mathrm{rad} / \mathrm{s}$, hasta que la muestra alcanzó $90^{\circ} \mathrm{C}$, manteniéndose constante durante $2,5 \mathrm{~min}$. Posteriormente, se aplicó una rampa de enfriamiento, bajo las mismas condiciones, hasta alcanzar $30^{\circ} \mathrm{C}$ (Enríquez et al. 2013).

Determinación de temperaturas de transición térmica de polímeros por Calorimetría de Barrido Diferencial (DSC). Se siguió la norma ASTM D3418-08 (2008); a 5-10mg de almidón, se les determinaron las temperaturas de transición de primer orden (fusión [Tm] y cristalización [Tc]) y a 10-20mg, se le determinó la temperatura de transición vítrea (Tg) (ASTM International, 2008).

Acondicionamiento del fruto. Los tomates, se lavaron por inmersión en agua potable, se desinfectaron con solución de hipoclorito de sodio (5ppm, 15min) y se secaron con toalla absorbente (Barco et al. 2011).

Preparación y aplicación del recubrimiento. Se realizó una pre-mezcla de los componentes del recubrimiento (agua, glicerol, 
almidón de yuca, aceite esencial, tween 80), a $300 \mathrm{rpm}$ y $40^{\circ} \mathrm{C}$, se calentó desde 40 hasta $75^{\circ} \mathrm{C}$ por 10 minutos hasta la gelatinización del almidón, durante 15min (Paz et al. 2016). El recubrimiento, se aplicó a temperatura ambiente con una esponja en condiciones asépticas, creando una capa uniforme en cada tomate.

Medición de variables. Cada 3 días, durante 15 días, se cuantificaron los parámetros: sólidos solubles totales, depositando una gota de jugo sobre el prisma del refractómetro digital (Castillo et al. 2018); firmeza en la máquina universal de ensayos por ensayo y error, hasta encontrar los parámetros de velocidad y de penetración índice de acidez y $\mathrm{pH}$, acidez titulable, usando el método volumétrico por neutralización; tasa de respiración, empleando el analizador de gas Oxybaby 6.0, que mide oxígeno residual y dióxido de carbono liberados por el fruto (Castillo et al. 2018); pérdida de peso por su diferencia (Castillo et al. 2018) y color, medido con colorímetro que usa el Sistema de coordenadas CIEL*a*b*, con los parámetros L (luminosidad), a* (variación color rojo-verde), b* (variación amarillo-azul), C (cromaticidad o saturación), h (ángulo Hue matiz o tono) (Castillo et al. 2018).

Diseño Experimental. Se utilizó un diseño factorial $4 x 6$ completamente al azar, donde los factores fueron "Tipo de recubrimiento", con 4 niveles (tratamientos Ti) y "Tiempo", con 6 niveles (Día 0, 3, 6, 9, 12 y 15), denominados PI, D3, D6, D9, D12, D15. Los niveles de tratamiento fueron:

T1 = Muestra Testigo (tomate sin recubrir);
T2 $=$ Recubrimiento con almidón de yuca modificado enzimáticamente;

T3= Recubrimiento con almidón de yuca modificado con ácido cítrico;

T4= Recubrimiento con almidón de yuca nativo.

Se realizaron tres réplicas con cuatro repeticiones por cada tratamiento, tomando 30 unidades experimentales por réplica. Los resultados, se evaluaron mediante pruebas paramétricas (ANOVA), pruebas no paramétricas y pruebas paramétricas con medidas repetidas; con una probabilidad de $95 \%$, empleando el software SPSS versión 19.

\section{RESULTADOS Y DISCUSIÓN}

Para la obtención del Equivalente de Dextrosa (E.D.), se siguió el protocolo planteado por Bonilla et al. (2014), obteniendo valores de absorbancia menores al rango $(0,2-0,6 \mathrm{~nm})$, que indican que no hubo E.D., por lo que se procedió a realizar las siguientes pruebas:

Caracterización reológica de las suspensiones de almidón. Se obtuvieron viscoamilogramas (Figura 1), de los cuales, se extraen resultados (mediante el software Data Analysis de TA Instruments), que permiten analizar el comportamiento de los almidones en cuanto a temperatura de inicio de gelatinización, viscosidad máxima, facilidad de cocción, inestabilidad del gel e índice de gelificación.

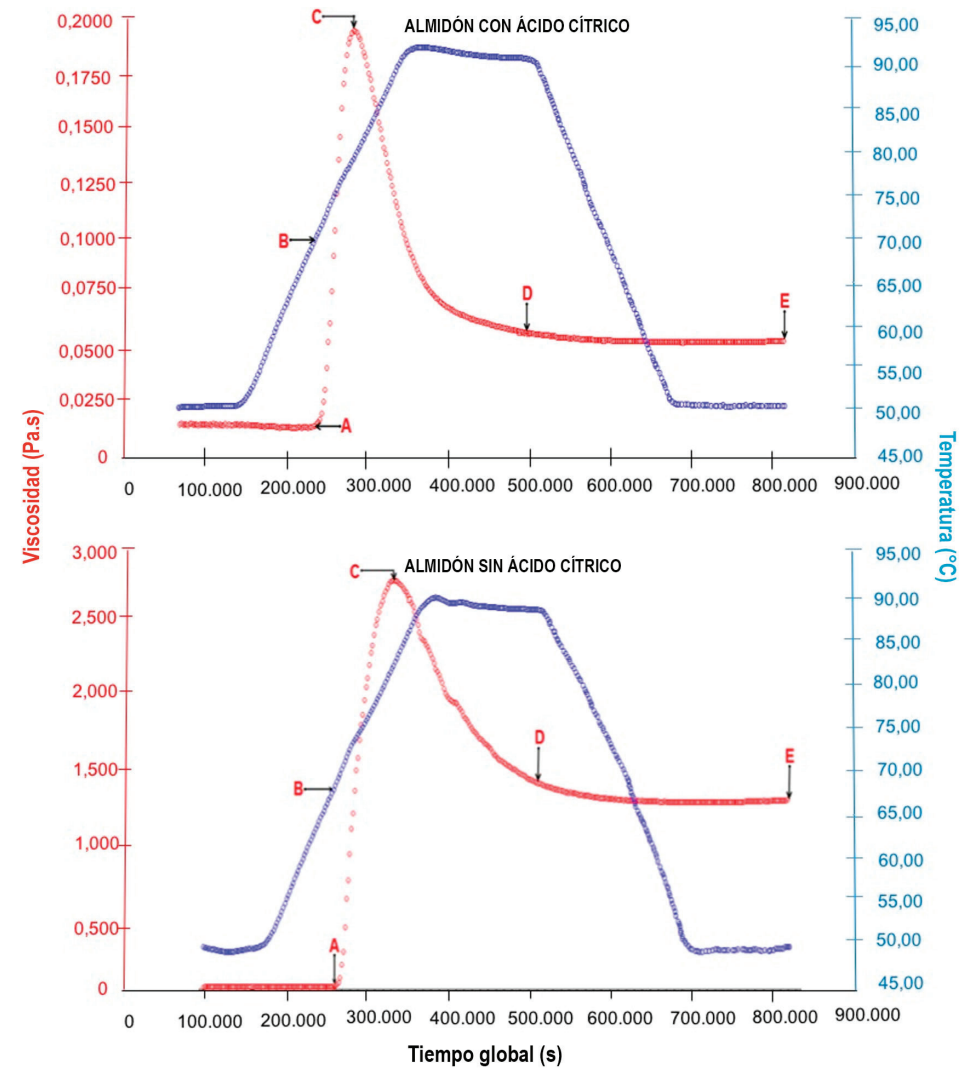

Figura 1. Viscoamilogramas del almidón modificado y sin modificar. A) Cambio brusco de viscosidad; B) Temperatura de inicio de gelatinización;C) Viscosidad máxima; D) Viscosidad mínima; E) Viscosidad final. 
Temperatura de inicio de gelatinización. El inicio de la gelatinización del almidón modificado con y sin ácido cítrico ocurrió a 252 y 259,012 s y temperaturas de 69,1 y $68,7^{\circ} \mathrm{C}$, respectivamente (Figura 1), indicando que el almidón modificado con ácido cítrico tardó menos en incrementar la viscosidad en comparación con el almidón sin ácido. El aumento de temperatura de gelatinización en los almidones modificados con ácido, se puede atribuir a que los cristales formados durante la retrogradación fueron más pequeños e imperfectos (Rivas et al. 2008).

Viscosidad. La viscosidad mínima del almidón sin ácido cítrico fue de 1,46, la final de 1,347 y la máxima de 2,913Pa*s; mientras que las del almidón modificado fueron 0,0538, 0,1925 y 0,04981 $\mathrm{Pa}^{*}$, respectivamente, debido, posiblemente, a que los gránulos de almidón modificado fueron atacados por el ácido durante el proceso de modificación, rompiéndolo (Méndez et al. 2009) y causando una disminución en el tiempo $(298,004 \mathrm{~s})$ y la temperatura de gelatinización $\left(78,4^{\circ} \mathrm{C}\right)$, al llegar a la máxima viscosidad. A $52^{\circ} \mathrm{C}$ (temperatura, en la que se realizó la modificación), posiblemente el almidón gelatinizó y, debido a eso, en el viscoamilograma (Figura 1), se observa que solo gelatinizaron los pocos gránulos que quedaron enteros, es decir, los residuos. Además, se observó que el almidón sin ácido cítrico necesitó mayor temperatura $\left(83,4^{\circ} \mathrm{C}\right)$ y mayor tiempo $(333,004 s)$ para alcanzar el punto máximo de viscosidad respecto al almidón modificado, lo que indica que requiere más energía, en el momento de realizar una gelatinización.

Facilidad de cocción. El almidón modificado con ácido cítrico presentó mayor facilidad de cocción, debido a que el tiempo transcurrido en alcanzar el hinchamiento de todos los gránulos desde el momento en que este se inicia, es menor $(46,004 \mathrm{~s})$ respecto al almidón sin ácido $(73,992 s)$. Este comportamiento del almidón modificado permite realizar una gelatinización más fácil, porque se logra en menor tiempo. De acuerdo con lo referenciado por Badui (2006), los valores de $\mathrm{pH}$ menores a 5 o mayores de 7 , tienden a reducir la temperatura de gelatinización y acelerar el proceso de cocción, lo que puede ser la razón del comportamiento del almidón modificado, con $\mathrm{pH}$ promedio de 1,7.

Inestabilidad del gel. La inestabilidad del gel del almidón con ácido cítrico fue de $0,1387 \mathrm{~Pa}^{*}$ s y la del almidón sin ácido fue de 1,453Pa*s; con esto, se podría aseverar que el almidón sin acidificar presentó menor estabilidad de la pasta. Quien presenta mayor inestabilidad del gel es el almidón con ácido cítrico, porque tiene un porcentaje de pérdida de viscosidad de 72,05 respecto al 49,88\% del almidón sin ácido cítrico (Figura 1). En este sentido, se puede afirmar que la viscosidad en el almidón modificado decayó, es decir, que aunque el almidón se gelatiniza, el producto que se forma es muy inestable, debido, posiblemente, a la acción del ácido cítrico sobre las zonas amorfas del almidón, fragmentando el gránulo (Méndez et al. 2009), con menor estabilidad de la pasta (gel débil), al realizarse la cocción (Badui, 2006).

Índice de gelificación. El índice de gelificación del almidón modificado con ácido cítrico es menor $\left(0,00399 \mathrm{~Pa}^{*} \mathrm{~s}\right)$ que el del almidón sin ácido $\left(0,113 \mathrm{~Pa}^{*} \mathrm{~s}\right)$. En conclusión, la modificación de almidón de yuca variedad SM 707-17 con ácido cítrico, permitió obtener un almidón con mayor facilidad de cocción, temperaturas de gelatinización más bajas, aunque presentó baja viscosidad y estabilidad del gel.

Temperatura de transición vítrea (Tg). La temperatura de transición vítrea $(\mathrm{Tg})$ para el almidón de yuca con ácido fue de $150,06^{\circ} \mathrm{C}$ y para el almidón sin ácido cítrico de $193,97^{\circ} \mathrm{C}$, valores superiores a la temperatura ambiente $\left(19^{\circ} \mathrm{C}\right)$, lo que significa que los almidones se comportan como materiales vítreos a temperatura ambiente (Enríquez et al. 2013). El almidón modificado con ácido cítrico presentó una $\mathrm{Tg}$ menor que el almidón sin ácido, comportamiento que se pudo deber a que el tratamiento ácido desorganiza parte de la estructura del almidón, disminuyendo su peso molecular.

Temperatura de fusión (Tm). El almidón modificado con ácido cítrico presentó menor valor de $\operatorname{Tm}\left(158,67^{\circ} \mathrm{C}\right)$ que el almidón sin ácido $\left(165,26^{\circ} \mathrm{C}\right)$, posiblemente, por el efecto del ácido sobre el rompimiento del gránulo, causando una disminución en la temperatura necesaria para fundir los cristales y alterar su estructura. La entalpía, se obtuvo a partir de los termogramas (Figura 2) usando, para ello, el software TA Universal Analysis, encontrando que para el almidón sin ácido fue mayor $\left(176,5 \mathrm{~J} \mathrm{~g}^{-1}\right)$ que para el almidón con ácido cítrico $\left(161 \mathrm{~J} \mathrm{~g}^{-1}\right)$, lo que indica que en el momento de fundir los gránulos, se requiere más energía para desorganizar las cadenas (Enríquez et al. 2013). Cuando se disminuye la entalpía o la fusión, el almidón se vuelve menos cristalino, porque aumentar la cristalinidad requiere más energía, es decir, el almidón sin ácido cítrico es más organizado que el modificado, haciendo que en la nueva organización molecular disminuyera la entalpía, pues para fundir un almidón es necesario romper la estructura semicristalina original (Villada et al. 2008).

Observación microscópica del gránulo de almidón de yuca para almidones nativo y modificado con ácido cítrico y enzimáticamente. Las micrografías con Microscopía Óptica de Alta Resolución (MOAR) revelaron que en el almidón nativo se pueden apreciar los bordes de los gránulos y pequeñas aglomeraciones esféricas, con hilum definido y céntrico en forma de asterisco, indicando que el almidón no está gelatinizado (Sívoli et al. 2009). El almidón modificado con ácido cítrico presenta alteraciones en su superficie, por formas y apariencias irregulares truncadas, como si tuviera cortes en diferentes direcciones; los gránulos, se notaron quebrados o fraccionados, debido a la combinación de temperatura y adición de ácido cítrico; cualquier modificación puede alterar la forma, la función y el comportamiento de la biomolécula. Se aprecian hendiduras más pronunciadas en los gránulos modificados con ácido cítrico, debido al rompimiento ocasionado por el ácido y la degradación de las regiones amorfas y cristalinas, fenómenos confirmados por estudios comparativos entre hidrólisis ácida y enzimática en almidones de tubérculos (Martín \& López, 2009).

Sólidos solubles totales (SST). El ANOVA no encontró diferencias significativas entre los tratamientos $(\mathrm{p}>0,05)$, para los factores Recubrimiento y Tiempo y la interacción entre ellos, lo que 


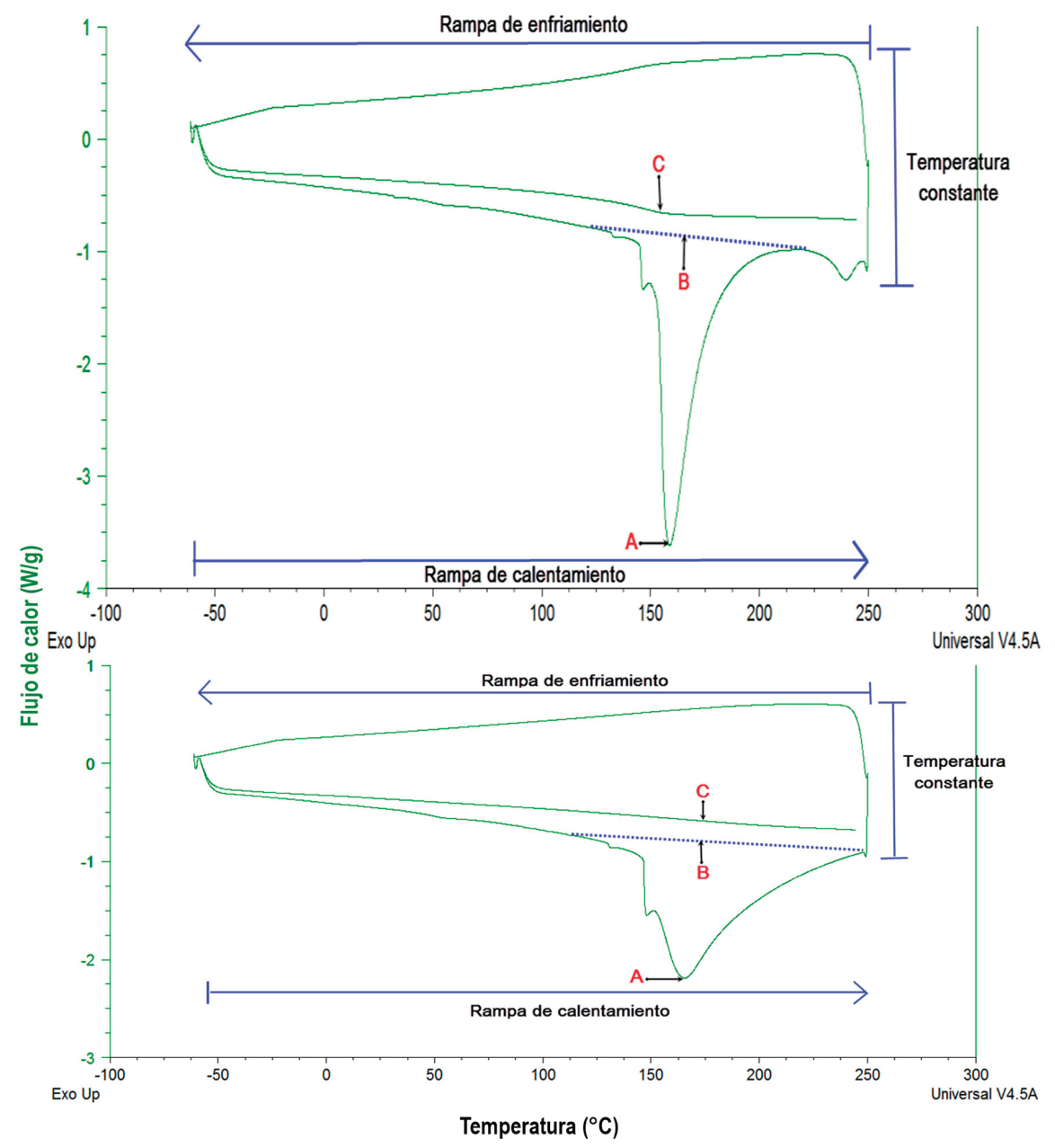

Figura 2. Termogramas de los almidones. A) almidón modificado con ácido cítrico; B) almidón sin modificación.

indica que este factor no incidió en los valores de sólidos solubles totales, ya que, estadísticamente, los recubrimientos son iguales (Figura 3), comportamiento que se ha encontrado en otros frutos, como banano (Barco et al. 2011), papaya (Castillo et al. 2018), mango, maracuyá y plátano (Torres et al. 2013).

Firmeza. Para el factor Recubrimiento, no se encontraron diferencias significativas entre los tratamientos ( $p>0,05)$, lo que indica que este factor no incidió en la firmeza de los frutos, ya que, estadísticamente, no existen efectos que presenten diferencia significativa entre los recubrimientos. Al evaluar la interacción Recubrimiento-Tiempo en el ANOVA, se registró que no hay relación entre ellos, es decir, tanto el tiempo como el tipo de recubrimiento actúan de forma independiente en los cambios de firmeza de los frutos. Castillo et al. (2018) hallaron diferencias significativas en aplicación de recubrimiento a base de almidón modificado por vía enzimática, resultado que puede obedecer a una estructura más sólida y ordenada de las partículas, al usar esta última.

Índice de acidez - pH. En lo que respecta al factor "Recubrimiento", mediante la prueba de Kruskal Wallis y por efectos de significancia, se determinó que no hubo cambios significativos $(p>0,05)$ en el $\mathrm{pH}$, que hayan sido producidos por el tipo de recubrimiento. En este caso, la relación $\mathrm{pH}$ - Tiempo es directamente proporcional en todos los tratamientos, es decir, a medida que avanza el tiempo, el $\mathrm{pH}$ se incrementa en los frutos.

Acidez titulable. La prueba de Kruskal Wallis determinó que el factor recubrimiento no incidió $(p>0,05)$ sobre el porcentaje de ácido cítrico encontrado, pero sí lo hizo sobre el tiempo ( $\mathrm{p}<$ 0,05), factores Recubrimiento y Tiempo, respectivamente, igual a lo obtenido por Castillo et al. (2018) y Torres et al. (2013). Se analizó con la prueba de comparaciones múltiples U de Mann Whitney, que determinó que la acidez titulable tuvo el mayor valor al inicio del estudio $(0,3535)$ y disminuyó progresivamente hasta el día 9 , con una pequeña recuperación el día 12 , donde tuvo un valor similar al del día 6, ubicándose estos dos días en un solo grupo, a diferencia de los demás donde hubo cambios estadísticamente significativos.

Tasa de Respiración. Se usó la prueba de medidas repetidas, usando, como criterio de decisión la significancia, que reportó que el porcentaje de $\mathrm{O}_{2}$ no se vio influenciado por el tiempo 


\section{Sólidos solubles totales ( ${ }^{\circ}$ Brix)}
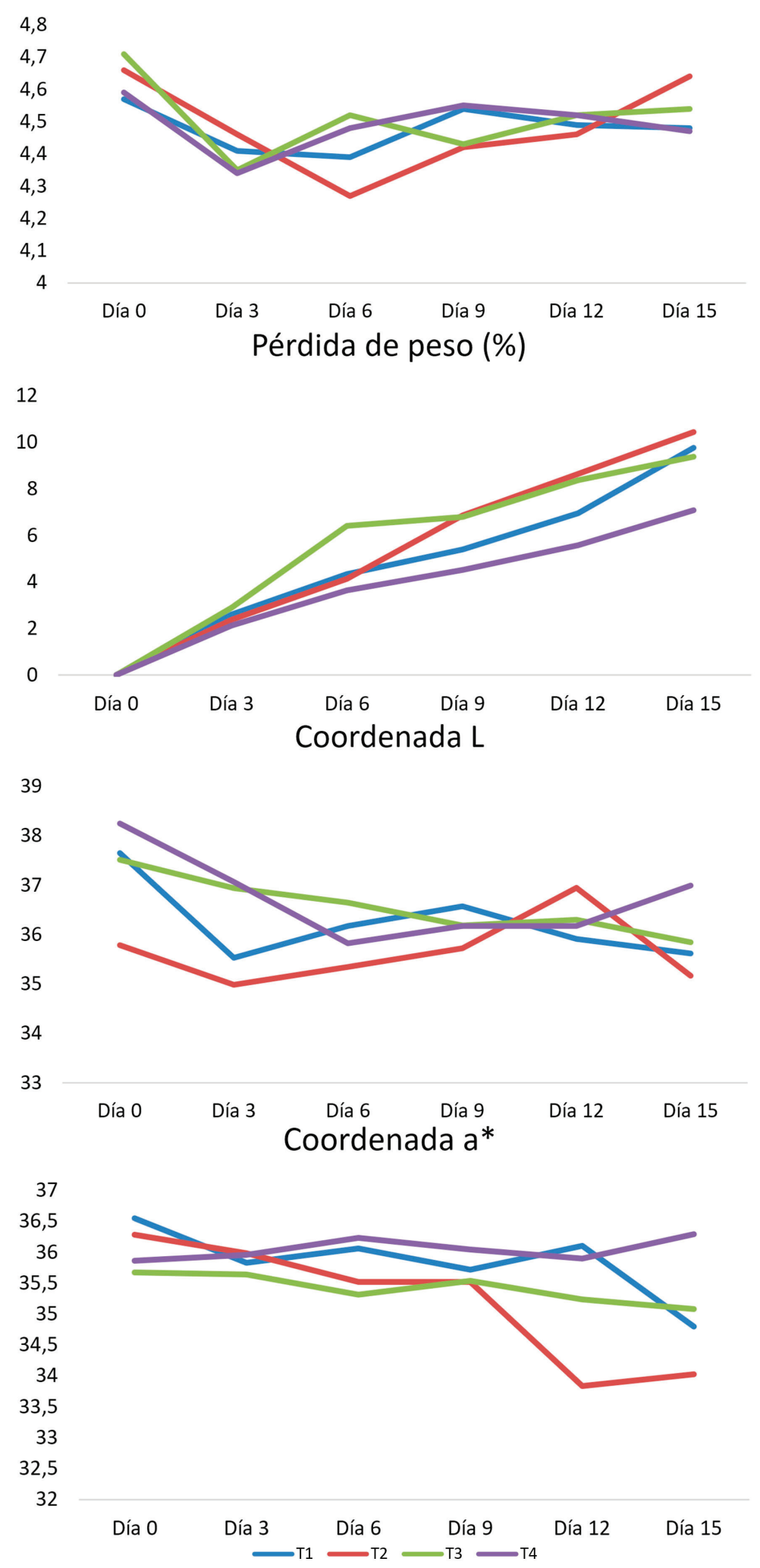

Figura 3. Comportamiento de los parámetros fisiológicos. 
( $p>0,05)$. De manera similar, el análisis estadístico demostró que el factor recubrimiento no afectó la variable en cuestión, ya que los tratamientos no presentaron diferencias estadísticamente significativas. El análisis demostró que no hay interacción entre Tiempo y Recubrimiento ( $\mathrm{p}>0,05$ ), lo que significa que el recubrimiento influyó de manera independiente sobre el tiempo. Dussan et al. (2014) indican que la utilización de ácido cítrico reduce la tasa de respiración y de producción de etileno, lo que concuerda con los hallazgos de este estudio.

En el porcentaje de $\mathrm{CO}_{2}$, la interacción entre tiempo y recubrimiento no fue significativa ( $\mathrm{p}>0,05)$, por lo que se puede inferir que la influencia del tiempo fue independiente de la influencia del recubrimiento. Respecto a la influencia del tipo de recubrimiento, el ANOVA, de medidas repetidas, muestra claramente que no hubo efecto $(\mathrm{p}>0,05)$ sobre el porcentaje de $\mathrm{CO}_{2}$; la prueba de Tukey para recubrimiento, resuelve que todos los tipos de recubrimientos ejercieron igual influencia sobre el contenido de $\mathrm{CO}_{2}$. Teniendo en cuenta que el estudio se realizó en tomates con grado de madurez 4 , se puede inferir que el recubrimiento que menos efecto tuvo en el retraso de la maduración fue T2, por su disminución en el consumo de $\mathrm{O}_{2}$ y el incremento en $\mathrm{CO}_{2}$ liberado, tal como lo mencionan Suárez et al. (2015), debido al estrés causado por la aplicación del recubrimiento; el tratamiento que retardó la maduración en sus frutos fue $\mathrm{T} 3$ por su consumo de $\mathrm{O}_{2}$ durante su desarrollo y menor liberación de $\mathrm{CO}_{2}$, lo que indica que, posiblemente, la enzima piruvato descarboxilasa tuvo menos efecto en los frutos de este tratamiento, retrasando así su senescencia.

Porcentaje de pérdida de peso (\% PP). El ANOVA mostró que en el factor "Tiempo" hay diferencias $(p<0,05)$, indicando que, a través del tiempo, el peso de los frutos cambió. La interacción entre los factores "Tiempo vs Recubrimiento" no es significativa ( $p>$ $0,05)$, por lo que se infiere que el tiempo y el recubrimiento afectan al fruto de forma independiente, analizándose el factor tiempo separado del factor recubrimiento; éste último no influyó ( $p>0,05)$ en la pérdida de peso de los frutos (Figura 3), por lo tanto, se infiere que al aplicar cualquier recubrimiento de los planteados, no se van a encontrar diferencias, comportamiento similar al encontrado por Chiumarelli et al. (2011), en muestras de mango pre tratadas.

Color: Los resultados obtenidos en las coordenadas L, a* y b* y los parámetros $\mathrm{C}$ y h, no presentaron diferencias significativas entre tratamientos y no existió interacción entre los factores Tiempo Recubrimiento", lo que indica que el recubrimiento, por sí solo, no incidió sobre la preservación del color del tomate y que todos los cambios generados en las variables de respuesta, sin excepción, fueron a causa del tiempo (Figura 3). Los recubrimientos no se adhirieron bien a la superficie del tomate, efecto que también presentó Gamboni et al. (2015) y que se atribuye a los enlaces débiles de las cadenas y sus fuerzas de adhesión y cohesión, así como también a la diferencia en la tensión superficial del recubrimiento y de la epidermis.
Agradecimientos: Los autores expresan agradecimiento a la Universidad del Cauca, por su aporte en el desarrollo del proyecto. Conflicto de intereses: El manuscrito fue preparado y revisado con la participación de todos los autores, quienes declaramos que no existe ningún conflicto de intereses que ponga en riesgo la validez de los resultados presentados. Financiación: Este estudio fue financiado por la Universidad del Cauca de Popayán (Cauca, Colombia).

\section{REFERENCIAS}

1. ALZATE, D.A.; MIER, G.; AFANADOR, L.; DURANGO, D.; GARCÍA, C. 2009. Evaluación de la fitotoxicidad y la actividad antifúngica contra Colletotrichum acutatum de los aceites esenciales de tomillo (Thymus vulgaris), limoncillo (Cymbopogon citratus) y sus componentes mayoritarios. Vitae (Colombia). 16(1):116-125.

2. AMERICAN SOCIETY FOR TESTING AND MATERIALS, ASTM INTERNATIONAL. 2008. Norma ASTM D341808. Estandard test method for transition temperatures and enthalpies of fusión and crystallization of polymer by Differential Scanning Calorimetry. Philadelphia, United State of America. 7p.

3. ASOCIACIÓN HORTOFRUTÍCOLA DE COLOMBIA, ASOHOFRUCOL, A. H. de C. 2014. Balance y perspectivas del sector hortifrutícola. Frutas \& Hortalizas, Rev. Asociación Hortifrutícola de Colombia (Colombia). 33(12):11-14

4. BADUI DERGAL, S. 2006. Química de los alimentos. Ed. Pearson education. (México). 716p.

5. BARCO, P.; BURBANO, A.; MOSQUERA, S.; VILLADA, H.; NAVIA, D. 2011. Efecto del recubrimiento a base de almidón de yuca modificado sobre la maduración del tomate. Rev. Lasallista de Investigación (Colombia). 8(2):96103.

6. BONILLA, J.R.; HOYOS, J.L.; VILLADA, H.S. 2014. Modificación enzimática de almidón de yuca Manibot esculenta Crantz) para el desarrollo de películas flexibles. Biotecnología en el sector agropecuario y agroindustrial (Colombia). 12(1):134-143.

7. CASTILLO, D.A.; SALAZAR, K.; MOSQUERA, S.A.; RENGIFO, E. 2018. Efecto de recubrimientos de almidón modificado de yuca, proteína aislada de soya y aceite esencial de orégano aplicados a la papaya. Rev. U.D.C.A. Act. \& Div. Cient. (Colombia). 21(1):71-80. https://doi. org/10.31910/rudca.v21.n1.2018.664

8. CHIUMARELLI, M.; FERRARI, C.; SARANTÓPOULOS, C.; HUBINGER, M. 2011. Fresh cut "Tommy Atkins" mango pre-treated with citric acid and coated with cassava (Manihot 
esculenta Crantz) starch or sodium alginate. Innovative food science and emerging technologies (United States of America). 12(3):381-387. https://doi.org/10.1016/j. ifset.2011.02.006

9. DUSSAN, S.; TORRES, C.; REYES, P. 2014. Efecto del recubrimiento comestible sobre los atributos fisicoquímicos de mango "Tommy Atkins" mínimamente procesado y refrigerado. Acta Agronómica (Colombia). 63(3):212-221. http://doi.org/10.15446/acag.v63n3.40973.

10. ENRÍQUEZ, M.; VELASCO, R.; FERNÁNDEZ, A. 2013. Caracterización de almidones de yuca nativos y modificados para la elaboración de empaques biodegradables. Biotecnología en el Sector Agropecuario y Agroindustrial (Colombia). 11(2):21-30.

11. GAMBONI, J.E.; SLAVUTSKY, A.M.; BERTUZZI, M.A. 2015. Estudio de las fuerzas de adhesión de películas de almidón sobre diferentes soportes. INIQUI (CONICET), CIUNSa, Facultad de Ingeniería (Universidad Nacional de Salta).11p.

12. GUTIÉRREZ, T.; PÉREZ, E.; GUZMÁN, R.; TAPIA, M.; FAMÁ, L. 2014. Physicochemical and functional properties of native and modified by crosslinking, dark-cush-cush yam (Dioscorea trifida) and Cassava (Manihot esculenta) starch. J. Polymer and Biopolymer Physics Chemistry (United States of America). 2(1):1-5. http://doi.org/10.12691/ jpbpc-2-1-1

13. INTERNATIONAL ASSOCIATION FOR CEREAL CHEMISTRY, ICC. 1995. ICC Standard Method No. 162. Rapid pasting method using the Newport rapid visco analyser. En: BASTIOLI, C. 2005. Handbook of biodegradable polymers: isolation, synthesis, characterization and applications. Rapra Technology Limited (United Kingdom). 549p.

14. MARTÍN, J.; LÓPEZ, E. 2009. Modificación física del almidón de yuca y evaluación de la susceptibilidad a la hidrólisis enzimática por una alfa amilasa. Rev. Colombiana de Química. 38(3):395-408.

15. MÉNDEZ, A.; MARTÍNEZ, F.; VÉLES, J.; MORENO, C.; DEL RÍO, J.; MORENO, E. 2009. Efecto de la adición de ácido cítrico sobre la degradación de las aflatoxinas y las propiedades funcionales de productos extrudidos de sorgo. Interciencia (Venezuela). 34(4):252-258.

16. ORDOÑEZ, D.; ZÚÑIGA, D.; HOYOS, J.; MOSQUERA, S.; MOSQUERA, L. 2014. Efecto de recubrimiento de almidón de yuca modificado y aceite de tomillo aplicado al pimiento (Capsicum annumm). Rev. Mexicana de Ciencias Agrícolas (México). 5(5):795-805.
17. PAZ, S.; MOSQUERA, S.; VILLADA, H. 2016. Evaluación de la permeabilidad de un recubrimiento elaborado a partir de almidón de yuca nativo y modificado. Vitae (Colombia). 23(1):222-225.

18. PÉREZ, V. 2017. World leader in carbohydrate ingredients. Almidones modificados. Disponible desde internet en: https://www.academia.edu/15338868/World_leader_in_ carbohydrate_ingredients_Almidones_modificados [con acceso el 12/12/2016]

19. RIOJAS, H.; GORTÁRES, P.; MONDACA, I.; BALDERAS, J. 2011. Aplicación de Tween 80 y D - Limoneno en la biorremediación de suelo contaminado por hidrocarburos. Ide@s CONCYTEG (México).6(71):571-584.

20. RIVAS, M.; MÉNDEZ, M.G.C.; SÁNCHEZ, M.M.; NÚÑEZ, M.C.; BELLO, L.A. 2008. Caracterización morfológica, molecular y fisicoquímica del almidón de plátano oxidado y lintnerizado. Agrociencia (México). 42(5):487-497.

21. SÁNCHEZ, D.; CONTRERAS, J.; NEVÁREZ, G.; AGUILAR, C. 2015. Caracterización de películas comestibles a base de extractos pécticos y aceite esencial de limón Mexicano. CyTA J. Food (México). 13:16-25. http://doi.org/10.1080 $/ 19476337.2014 .904929$

22. SAN MARTIIN, C.; ORDAZ, V.; SÁNCHEZ, P., BERYL, M.; BORGES, L. 2012. Calidad de tomate (Solanum lycopersicum L.) producido en hidroponia con diferentes granulometrías de tezontle. Agrociencia (Uruguay). 46(3):243-254.

23. SÍVOLI, L.; PÉREZ, E.; RODRÍGUEZ, P.; RAYMÚNDEZ, M.; AYESTA, C. 2009. Técnicas microscópicas y de dispersión de luz empleadas en la evaluación de la estructura del almidón nativo de yuca (Manihot esculenta $\mathrm{C}$ ). Acta Microscópica (Venezuela). 18(3):195-203.

24. SUÁREZ, A.; TREJO, A.; LIRA, M. 2015. Desarrollo de un recubrimiento con efecto antifúngico y antibacterial a base de aceite esencial de orégano para conservación de papaya "maradol". Revista Iberoamericana de Tecnología Pos cosecha (España).16(1):58-63.

25. TORRES, R.; MONTES, E.; PÉREZ, O.; ANDRADE, R. 2013. Relación del color y del estado de madurez con las propiedades fisicoquímicas de frutas tropicales. Información Tecnológica (Chile). 24(3):51-56.

26. VILLADA, H.; ACOSTA, H.; VELASCO, R. 2008. Investigación de Almidones Termoplásticos, Precursores de Productos 